\title{
Permeability through Cellulose Membranes Grafted with Vinyl Monomers in a Homogeneous System I. Diffusive Permeability through Acrylonitrile Grafted Cellulose Membranes
}

\author{
Noboru NishioKA, Keiji WatASE, Keiji Arimura, \\ Kouichi KoSAI, and Masakuni UNO* \\ Faculty of Engineering and *Junior College Division, \\ Osaka Electro-Communication University, \\ Neyagawa, Osaka 572, Japan
}

(Received April 3, 1984)

\begin{abstract}
The homogeneous graft copolymerization of acrylonitrile onto cellulose was carried out in a dimethyl sulfoxide (DMSO)-paraformaldehyde solvent system. Three kinds of membranes were prepared: higher grafting efficiency membranes (H-GE membrane), lower grafting efficiency membranes (L-GE membrane), and blended membranes. The diffusive permeability of solutes through the water-swollen membranes was investigated. The permeability and microphase separated structure depended remarkably on the kind of membrane. Permeability through the $\mathrm{H}$ GE membranes increased with increasing polyacrylonitrile content and leveled off, but that through the L-GE and blended membranes decreased. Permeability through the H-GE membranes was superior to that through the cellulose membrane cast from the DMSO solution of cellulose. However, the good permeability through the H-GE membranes could not be explained by the difference in water content of membranes.
\end{abstract}

KEY WORDS Homogeneous Grafting / Cellulose / Acrylonitrile / Grafted Membrane / Blended Membrane / Diffusive Permeability / Microphase Separated Structure / Water Content /

The commercial regenerated cellulose membrane, Cuprophan, produced by the cuprammonium process has been widely used as a hemodialysis membrane owing to its good solute permeability and mechanical strength. However, it is also recognized that the diffusive permeability of solutes of molecular weight ranging from hundreds to thousands is not so good.

We may reasonably assume that the diffusive permeation of water soluble solutes proceeds only through the water phase in water-swollen membranes. ${ }^{1-3}$ Therefore, the water content of membranes is a significant parameter governing the diffusive permeation of solutes. However, the increase in water content usually causes a decrease in the mechanical strength of the membranes. To produce membranes of good permeability and mechanical strength, many studies have been carried out on amphiphilic copolymer membranes having the proper composition of hydrophilic and hydrophobic regions which function to control solute permeation and support the membrane, respectively. ${ }^{4-10}$

In this work, cellulose which has high mechanical strength in a water-swollen state was used as a hydrophilic membrane substrate. Vinyl monomers were grafted onto cellulose in a homogeneous system. Grafting onto cellulose is usually carried out in a heterogeneous system because of the lack of relevant solvent systems for cellulose. ${ }^{11,12}$ Grafting in a heterogeneous system proceeds in amorphous re- 
gions and/or on membrane surfaces. In a homogeneous solution system, cellulose dissolves molecularly so as to perform its role in grafting. ${ }^{13-16}$ In the previous paper, ${ }^{14-16}$ among the non-aqueous solvents for cellulose, dimethyl sulfoxide (DMSO) containing paraformaldehyde (PF) was chosen as a grafting medium because it is a nondegrading solvent for cellulose. ${ }^{17}$ The homogeneous grafting of vinyl monomers onto cellulose was carried out under various conditions to investigate the relationship between grafting conditions and the structures of the graft copolymers obtained.

The diffusive permeability through cellophanes grafted with vinyl monomers in a heterogeneous system has been already investigated. ${ }^{18,19}$ However, characterization of the graft copolymers obtained has not been carried out thoroughly. In this work, the wellcharacterized cellulose membranes grafted with acrylonitrile (AN) in the DMSO-PF solvent system were used and the diffusive permeability of various solutes through the AN grafted cellulose membranes of various compositions was investigated for the first time.

\section{EXPERIMENTAL}

\section{Grafting and Membrane Preparation}

The homogeneous grafting of AN onto cellulose in the DMSO-PF solvent system was carried out by the method described previously. ${ }^{14,16}$

The grafting mixtures were cast at a room temperature on glass plates and the DMSO and unreacted $\mathrm{AN}$ were allowed to evaporate at about $40^{\circ} \mathrm{C}$ under reduced pressure for a given period prior to the coagulation of the membranes in a water bath. After being immersed in water for a few weeks, the membranes were easily removed from the glass plates.

The DMSO solutions of cellulose and polyacrylonitrile (PAN) were mixed to give proper blended ratios. The blended mem- branes were also prepared by the same method as described above. The viscosity-average molecular weights, $M_{v}$, of PAN and cellulose used for the blended membranes were estimated to be $17 \times 10^{4}$ and $16 \times 10^{4}$ from the intrinsic viscosities, $[\eta]$, obtained with dimethylformamide (DMF) at $25^{\circ} \mathrm{C}^{20}$ and cadoxen at $20^{\circ} \mathrm{C},{ }^{21}$ respectively. All membranes were stored in distilled water until use.

An aliquot of each grafting mixture was poured into distilled water vigorously stirred to precipitate the graft product. Characterization of the graft products was carried out by the method described previously, ${ }^{14,15}$ and the results are summarized in Table I. Three series of membranes were prepared: a series of higher grafting efficiency membranes (H-GE membrane), a series of lower grafting efficiency membranes (L-GE membrane), and a series of blended membranes. The grafting efficiency (GE), a parameter characterizing graft products, expresses the ratio of the weight of graft polymer to that of polymer formed. It should be noted that the grafted membranes used contain the homopolymer component, homo-PAN.

\section{Diffusive Permeability}

A glass cell consisting of two compartments $\left(150 \mathrm{~cm}^{3}\right.$ each) was used. A membrane was clamped between the two compartments using suitable supporting and sealing devices. One compartment was filled with distilled water, and the other, with a solution of a permeant. Both compartments were "stirred during measurement of permeability and kept at $30 \pm 0.05^{\circ} \mathrm{C}$ in a water bath.

The diffusive permeability coefficient, $P$ (in $\mathrm{cm}^{2} \mathrm{~s}^{-1}$ ), was calculated according to the equation:

$$
P=-\frac{V(t) L}{2 S t} \ln \frac{\left(1-C_{2}(t) / C_{1}(t)\right)}{\left(1+C_{2}(t) / C_{1}(t)\right)}
$$

where $V(t)$ is the volume of the solution at time $t, L$, the membrane thickness, $S$, the effective membrane area $\left(3.14 \mathrm{~cm}^{2}\right)$, and $C_{1}(t)$ and 
Permeability through AN Grafted Cellulose Membranes

Table I. Characterization of grafted and blended membranes

\begin{tabular}{|c|c|c|c|c|c|c|}
\hline \multirow{2}{*}{ Sample code } & \multirow{2}{*}{$\frac{\mathrm{GE}}{\%}$} & \multicolumn{3}{|c|}{ PAN content $/ w^{\circ} / \%$} & \multirow{2}{*}{$\begin{array}{c}M_{v} \times 10^{-4} \\
\text { of } \text { graft }^{\mathrm{a}}\end{array}$} & \multirow{2}{*}{$\begin{array}{c}\text { Number of } \\
\text { grafts }\end{array}$} \\
\hline & & Overall & Graft-PAN & Homo-PAN & & \\
\hline \multicolumn{7}{|c|}{ H-GE Membrane } \\
\hline g-PAN 1 & 74.9 & 12.6 & 10.0 & 2.6 & 1.5 & 1.2 \\
\hline g-PAN 2 & 90.8 & 33.9 & 30.8 & 3.1 & 3.0 & 2.6 \\
\hline g-PAN 3 & 96.2 & 40.5 & 39.0 & 1.5 & 3.3 & 3.2 \\
\hline \multicolumn{7}{|c|}{ L-GE Membrane } \\
\hline g-PAN 11 & 11.0 & 17.9 & 2.0 & 15.9 & 2.0 & 0.2 \\
\hline g-PAN 12 & 10.0 & 26.1 & 2.6 & 23.5 & 1.2 & 0.5 \\
\hline g-PAN 13 & 10.5 & 38.9 & 4.0 & 34.9 & 3.0 & 0.2 \\
\hline g-PAN 14 & 11.0 & 61.9 & 6.8 & 55.1 & 0.9 & 1.2 \\
\hline \multicolumn{7}{|c|}{ Blended membrane ${ }^{b}$} \\
\hline b-PAN 1 & 0 & 10.0 & 0 & 10.0 & & \\
\hline b-PAN 2 & 0 & 20.0 & 0 & 20.0 & & \\
\hline b-PAN 3 & 0 & 30.0 & 0 & 30.0 & & \\
\hline b-PAN 4 & 0 & 40.0 & 0 & 40.0 & & \\
\hline b-PAN 5 & 0 & 50.0 & 0 & 50.0 & & \\
\hline
\end{tabular}

${ }^{\text {a }}$ Estimated from $[\eta]$ obtained in DMF at $25^{\circ} \mathrm{C} .{ }^{20}$

b The values of $M_{v}$ for cellulose and PAN are $16 \times 10^{4}$ and $17 \times 10^{4}$, respectively.

$C_{2}(t)$, the concentrations of the concentrate and diluent compartments at time $t$, respectively. The concentrations of both compartments were determined using $5 \mathrm{~cm}^{3}$ of the solutions picked up from both compartments simultaneously at certain time intervals. The logarithmic term of eq 1 was plotted against time and $P$ was determined from the slope.

Seven water soluble solutes of different molecular weight were used as permeants. Three samples of commercial poly(ethylene glycol) (PEG) of different molecular weight were fractionally precipitated at $30^{\circ} \mathrm{C}$ with toluene as the solvent and $n$-hexane, the precipitant. ${ }^{22}$ The middle fraction of each PEG sample was used as the permeant. The values of $M_{v}$ for PEG samples were estimated from $[\eta]$ obtained in benzene at $25^{\circ} \mathrm{C}^{23}$ The concentrations of various permeants were determined by the methods summarized in Table II.

\section{Hydraulic Permeability}

The hydraulic permeability of water, $K_{w}$, was measured with a reverse osmosis batch cell (Fujii Seiki FMD 300) having a volume of
Table II. Determination of solute concentrations

\begin{tabular}{lrl}
\hline \multicolumn{1}{c}{ Solute } & $\begin{array}{r}\text { Molecular } \\
\text { weight }\end{array}$ & \multicolumn{1}{c}{ Detection } \\
\hline $\mathrm{NaCl}$ & 58 & Titration with $\mathrm{AgNO}_{3}$ aq \\
Uric acid & 168 & Absorbance $(293 \mathrm{~nm})$ \\
Raffinose & 504 & Differential refractometer \\
Vitamine $\mathrm{B}_{12}$ & 1357 & Absorbance (570 nm) \\
PEG-IV & $2900^{\mathrm{a}}$ & Differential refractometer \\
PEG-VI & $8400^{\mathrm{a}}$ & Differential refractometer \\
PEG-XX & $38000^{\mathrm{a}}$ & Differential refractometer \\
\hline
\end{tabular}

${ }^{\text {a }}$ Estimated from $[\eta]$ obtained with benzene at $25^{\circ} \mathrm{C}^{23}$

$300 \mathrm{~cm}^{3}$ and an effective membrane area of $24.6 \mathrm{~cm}^{2}$.

\section{Water Content}

The membranes were immersed in distilled water for several days and the water on the membrane surfaces was blotted off prior to weighing. This blotting process was repeated several times so as to plot the weight of the water-swollen membrane against the number of times of blotting. The weights of the waterswollen membranes were determined by extrapolating the linear part of the curves to zero 
time. The weights of the dry membranes were determined by drying them to constant weight at about $100^{\circ} \mathrm{C}$. The water content was expressed as the ratio of the weight of water in the water-swollen membrane to that of the dry membrane.

\section{Electron Microscopy}

Cross sections of the membranes were observed with a scanning electron microscope (SEM), Shimadzu ASM-ST. The crosssections were obtained by fracturing the membranes in liquid nitrogen followed by coating with gold by vacuum deposition.

Transmission electron microscope (TEM) observation was conducted with a Hitachi HU-11A microscope. The membranes were stained by immersion in a $2 \%$ osmic acid aqueous solution for one or two days. The copolymers of $n$-butyl methacrylate and methyl methacrylate $(9: 1$ or $8: 2$ by weight) were used as embedding media. The embedded membranes were crosssectioned using a Sorball MT-2 ultramicrotome at a thickness of approximately $500 \AA$.

\section{Accessibility}

The membranes were dried over $\mathrm{P}_{2} \mathrm{O}_{5}$ under reduced pressure for $24 \mathrm{~h}$, immersed in $\mathrm{D}_{2} \mathrm{O}$ for $4 \mathrm{~h}$, and then dried again under reduced pressure for $24 \mathrm{~h}$. The infrared spectra were measured by placing the samples between $\mathrm{NaCl}$ plates with hexachlorobutadiene. The accessibility, $A$, was calculated according to the equation: ${ }^{24}$

$$
A=100 /\left(1+1.11 \times \frac{A_{\mathrm{OH}}}{A_{\mathrm{OD}}}\right)
$$

where $A_{\mathrm{OH}}$ and $A_{\mathrm{OD}}$ are the absorbances of $3360 \mathrm{~cm}^{-1}$ and $2530 \mathrm{~cm}^{-1}$, respectively.

\section{RESULTS AND DISCUSSION}

The membrane preparation was made by evaporating the casting solvent, DMSO, under reduced pressure at a given temperature be-

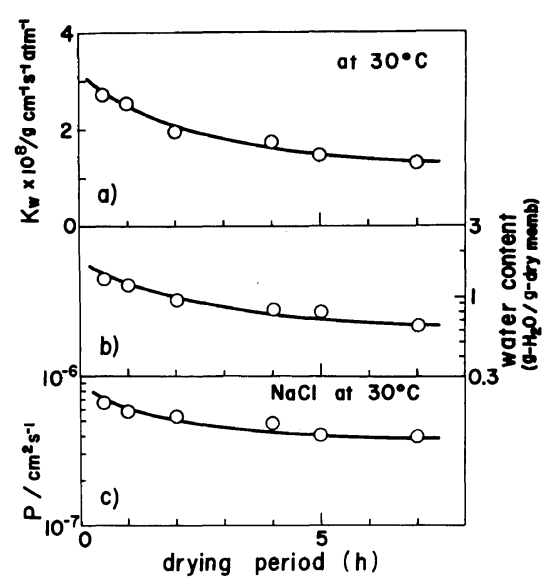

Figure 1. Effect of drying period on permeation characteristics: (a) hydraulic permeability of water at $30^{\circ} \mathrm{C}$ under $4 \mathrm{~kg} \mathrm{~cm}^{-2}$ pressure; (b) water content; (c) diffusive permeability of $\mathrm{NaCl}$ at $30^{\circ} \mathrm{C}$.

cause of the hygroscopicity and higher boiling temperature of DMSO. The effect of the drying period on the hydraulic permeability of water, water content, and diffusive permeability of $\mathrm{NaCl}$ is shown in Figure 1. All these permeation characteristics decrease with increasing drying period, indicating that the membrane gradually becomes dense. The drying period was determined as one day so that the membrane performance would have sufficient stability.

Figure 2 shows the SEM micrographs of fractured face for membranes subjected to different drying periods. Not all the membranes have skin layers and cavities, indicating that they are not porous but homogeneous symmetrical membranes.

The solute molecular weight dependence of the diffusive permeability for various membranes is illustrated in Figure 3. Permeability through each membrane decreases with increasing molecular weight of the permeant and two tendencies are apparent. Permeability through the Cuprophan membrane is several times superior to that through the membrane cast from the DMSO solution of cellulose, designated as the cellulose membrane, but the molecular weight dependence for both mem- 

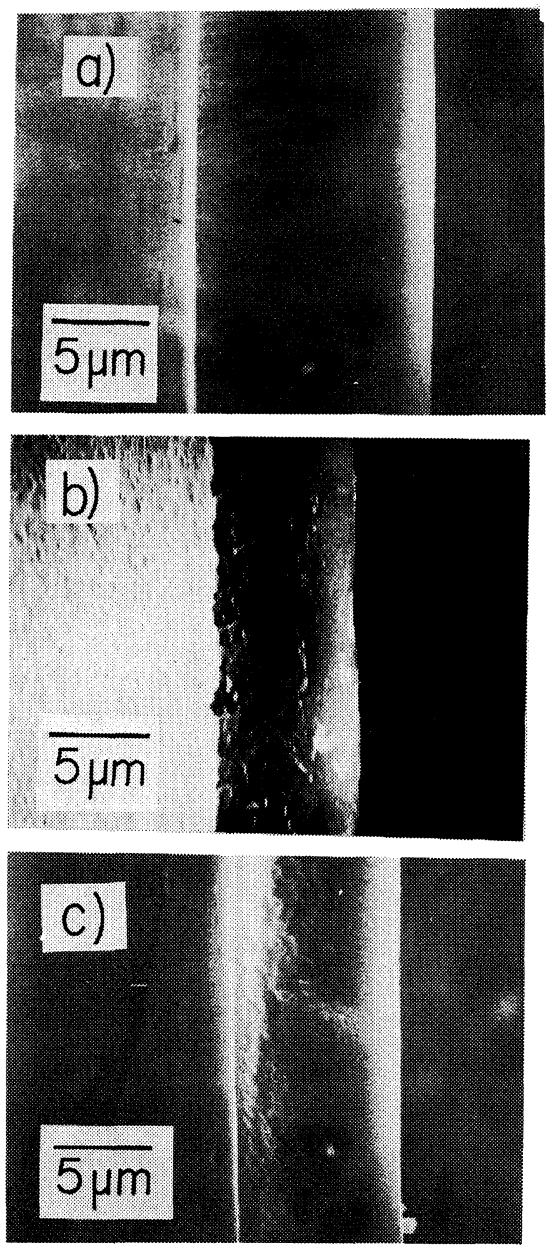

Figure 2. Scanning electron micrographs of fractured face for membranes of different drying periods: drying period; (a) $0.5 \mathrm{~h}$; (b) $1.0 \mathrm{~h}$; (c) $7.0 \mathrm{~h}$.

branes is similar. This seems reasonable since both membranes were made of cellulose though the preparation process was different.

The permeability of the lower molecular weight solutes through the g-PAN 3 membrane, an H-GE membrane, shows a more gradual dependence on molecular weight than that through the cellulosic membranes. It should be noted that permeability through the g-PAN 3 membrane is superior to that through the cellulose membrane over the molecular weight range investigated. Furthermore, the permeabilities of uric acid, raffinose, and vi-

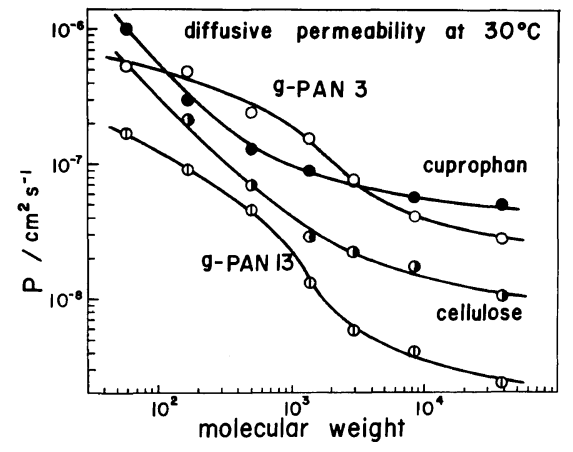

Figure 3. Molecular weight dependence of diffusive permeability for various membranes: (O) Cuprophan membrane; (O) cellulose membrane; (O) g-PAN 3 membrane; (D) g-PAN 13 membrane.

tamine $\mathrm{B}_{12}$ through the g-PAN 3 membrane are superior to those through the Cuprophan membrane. The difference in molecular weight dependence of the permeability for the cellulosic and AN grafted membranes may imply a difference in the interaction between the permeating solutes, membrane substrates, and water in the membranes. It has been reported that, for example, solutes containing hydrophilic groups influence transport properties through water-swollen membranes. ${ }^{25-29}$

Permeability through the g-PAN 13 membrane, an L-GE membrane, is several times less than that through the g-PAN 3 membrane, but both molecular weight dependences of the permeability are similar. In Table I, the PAN content for the g-PAN 3 and g-PAN 13 membranes is about $40 \%$ but the GE values for both membranes differ remarkably.

Figures 4 and 5 show the effect of PAN content on diffusive permeability for various solutes. The data for the Cuprophan and cellulose membranes are also indicated. The PAN content dependence of the permeability for each solute varies remarkably with the kind of membrane. The permeabilities of various solutes, except for $\mathrm{NaCl}$, through the $\mathrm{H}-\mathrm{GE}$ membranes increase with increasing PAN content and level off. It is of interest that the permeability of $\mathrm{NaCl}$ indicates the different 


\section{N. NisHIOKA et al.}

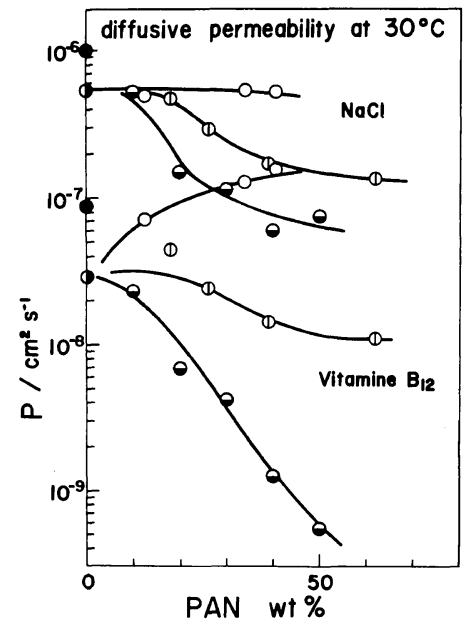

Figure 4. PAN content dependence of diffusive permeability for $\mathrm{NaCl}$ and vitamine $\mathrm{B}_{12}$ : (O) Cuprophan membrane; (๑) cellulose membrane; $(O)$ H-GE membrane; (D) L-GE membrane; $(\Theta)$ blended membrane.

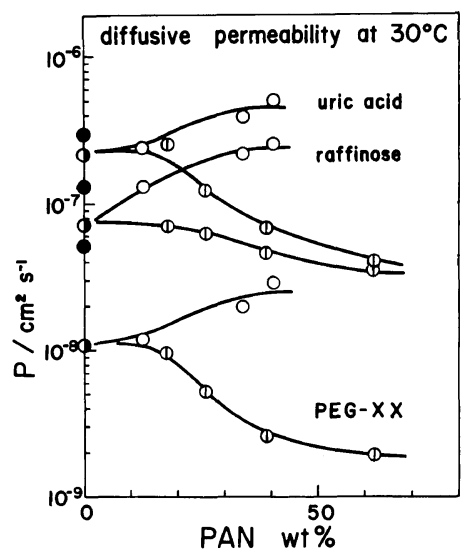

Figure 5. PAN content dependence of diffusive permeability for uric acid, raffinose, and PEG-XX: The symbols are the same as those in Figure 4.

dependence, suggesting a specific interaction between $\mathrm{NaCl}$ and the membranes. The permeabilities of all solutes investigated through the L-GE membranes decrease with increasing PAN content. The permeabilities of $\mathrm{NaCl}$ and vitamine $B_{12}$ through the blended membranes decrease more sharply than those through the L-GE membranes, indicating that, as shown in Figure 3, the difference in grafting efficiency influences the membrane performance sig-
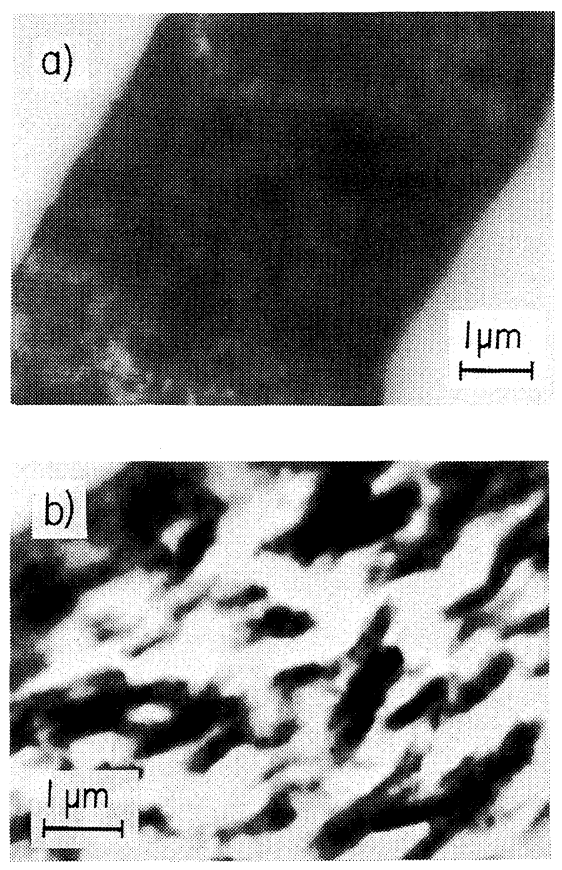

Figure 6. Transmission electron micrographs of the $\mathrm{H}$ GE membranes stained with osmic acid: (a) g-PAN 1; (b) g-PAN 2.

nificantly.

Graft copolymers have microphase separated structures due to the incompatibility of constituent polymers. These microdomain structures depend on such factors as copolymer composition, the molecular weights of constituent polymers, number of grafts, casting solvent, and so on. ${ }^{30}$ In the case of our grafted membranes, grafting efficiency would influence the microphase separated structures remarkably since, as indicated in Table I, the membranes contain the homo-PAN component. Furthermore, samples whose number of grafts is less than unity contain three components: cellulose, PAN, and AN grafted cellulose.

Figure 6 shows the TEM micrographs of the H-GE membranes. The dark domains indicate the cellulose phases stained with osmic acid. The H-GE membranes show fine microphase separated structures and the interfaces between the cellulose and PAN domains are not 

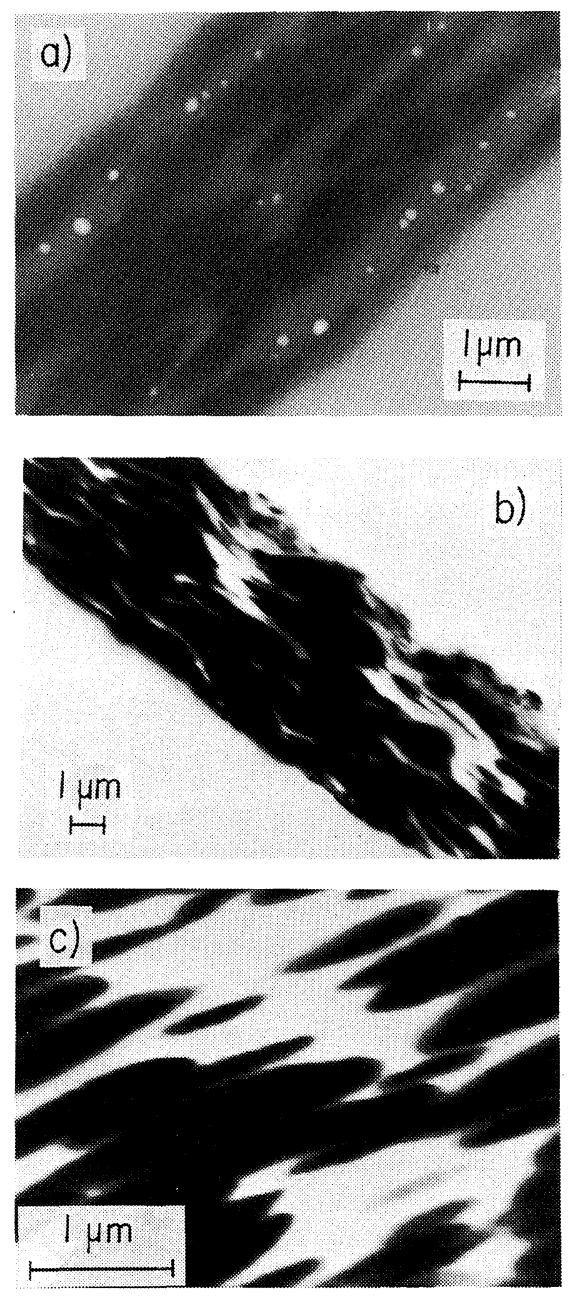

Figure 7. Transmission electron micrographs of the LGE membranes stained with osmic acid: (a) g-PAN 12; (b) g-PAN 13; (c) g-PAN 14.

clear. In Figure 7, the microphase separated structures of the L-GE membranes are clearer than those of the H-GE membranes, and the cellulose phases are dispersed in the continuous phase of PAN. This is probably because DMSO is a poor solvent for cellulose. Figure 8 shows the TEM micrographs of the blended membranes. The cellulose phases are dispersed in the continuous phase of PAN, similar to those of the L-GE membranes. However, the microphase separated structures of the blended membranes differ slightly from
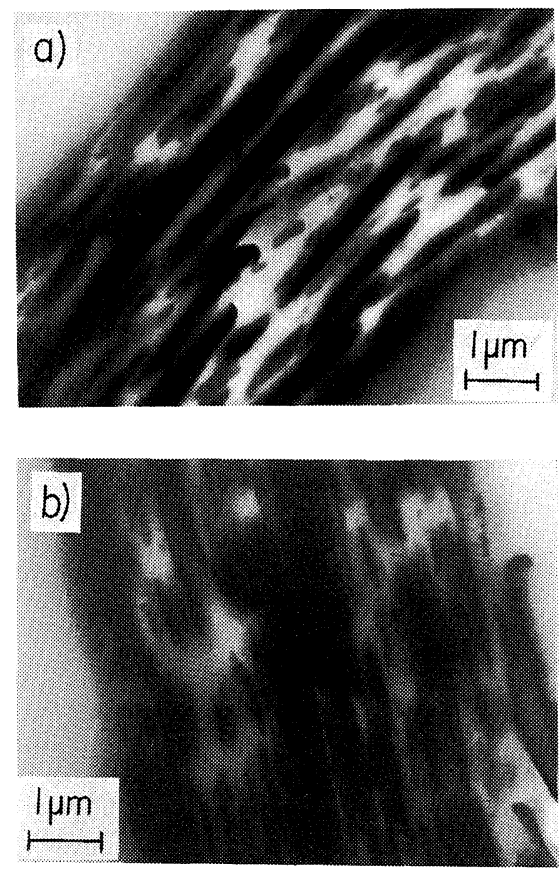

Figure 8. Transmission electron micrographs of blended membranes stained with osmic acid: (a) bPAN 1; (b) b-PAN 3.

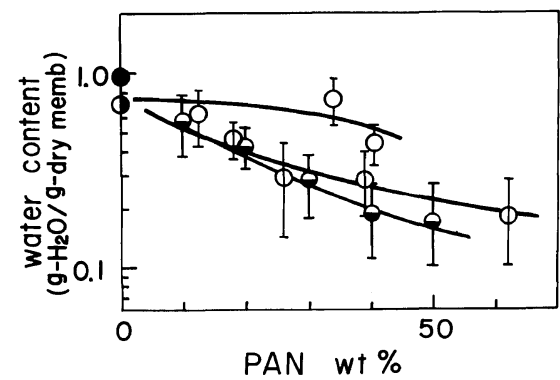

Figure 9. PAN content dependence of water content for various membranes: The symbols are the same as those in Figure 4.

those of the L-GE membranes. The lengths of the cellulose domains in the blended membranes seem longer than those in the L-GE membranes.

Factors governing the diffusive permeability of solutes are not only the membrane structures but also the water content of the membranes, interaction of the solutes with membrane substrates, and so on. ${ }^{31}$ Based on the 
free volume theory of diffusion, Yasuda et $a l .^{1-3}$ indicated that the diffusive permeability of solutes through membranes can be well explained by their water content. A similar treatment has also been reported for other membrane-solute systems. ${ }^{17,32,33}$

Figure 9 shows the relationship between water content and PAN content. The water content of the Cuprophan membrane is more than that of the cellulose membrane, as consistent with the superiority of permeability through the Cuprophan membrane to that through the cellulose membrane and the higher accessibility of the Cuprophan membrane than that of the cellulose membrane. The water content of the $\mathrm{H}-\mathrm{GE}$ membranes is nearly equal to that of the cellulose membrane and decreases only slightly with increasing PAN content. As shown in Figure 6, the microphase separated structures for the H-GE membranes are very fine. The presence of PAN chains thus hinder the crystallization of cellulose chains, causing the fraction of free hydroxy groups to increase. In Table III, this expectation is confirmed by the greater accessibility of the H-GE membranes compared to that of the cellulosic membranes. This explains that, in spite of the incorporation of hydrophobic PAN, the water content for the $\mathrm{H}$ GE membranes hardly depends at all on PAN content.

The water content for the L-GE and blended membranes decreases gradually. In these cases, the presence of PAN chains scarcely influences the crystallization of cellulose chains since, as evident from Figures 7 and 8, the interfaces between the cellulose and PAN phases are definite and the cellulose phases are surrounded by the continuous phase of PAN. The hydrophobicity of PAN remarkably influences both accessibility and water content.

As indicated above, permeability through the H-GE membranes is superior to that through the cellulose membrane, but the water content of the $\mathrm{H}-\mathrm{GE}$ membranes does
Table III. Accessibility determined by deuteration method for cellulosic and grafted membranes

\begin{tabular}{lc}
\hline & Accessibility \\
\cline { 2 - 2 } Sample code & $\%$ \\
\hline Cuprophan & 42.5 \\
Cellulose & 38.8 \\
g-PAN 2 & 51.5 \\
g-PAN 3 & 53.6 \\
g-PAN 11 & 18.3 \\
g-PAN 12 & 20.5 \\
g-PAN 13 & 29.0 \\
\hline
\end{tabular}

not differ significantly from that of the cellulose membrane. Moreover, the permeabilities of $\mathrm{NaCl}$ and vitamine $\mathrm{B}_{12}$ through the L-GE membranes differ remarkably from those through the blended membranes, whereas the water content for both membranes differ only slightly. The difference in permeability cannot be explained by that in total water content. It is well established that water molecules in membranes exist in many different states. ${ }^{34}$ Among them, bound water, i.e., water molecules bound to the polymer molecules through hydrogen bonding, is considered to reject solutes. ${ }^{35,36}$ On the other hand, it has been reported ${ }^{37}$ that bound water can be classed as that containing solutes and that devoid of them. Thus, it is important to estimate not only total water content but also the fraction of water associated with the permeation of solutes. Many factors govern the permselectivity of membranes and in our next paper we shall discuss the states of water in membranes in relation to the permeability of solutes through membranes. ${ }^{38}$

Acknowledgement. The authors are indebted to Dr. M. Nishii of Osaka Laboratory for Radiation Chemistry, Japan Atomic Energy Research Institute, for carrying out the SEM observations. 


\section{REFERENCES}

1. H. Yasuda, C. E. Lamaze, and L. D. Ikenberry, Makromol. Chem., 118, 19 (1968).

2. H. Yasuda, L. D. Ikenberry, and C. E. Lamaze, Makromol. Chem., 125, 108 (1969).

3. H. Yasuda, A. Peterlin, C. K. Colton, K. A. Smith, and E. W. Merrill, Makromol. Chem., 126, 177 (1969).

4. D. J. Lyman and B. H. Loo, J. Biomed. Mater. Res., 1, 17 (1967).

5. S. K. Brauman and B. K. Fritzinger, J. Appl. Polym. Sci., 16, 2439 (1972).

6. Y. Sakai, S. Hosaka, H. Tanzawa, and M. Itoga, Kobunshi Ronbunshu, 34, 801 (1977).

7. S. Yamashita, K. Takamura, Y. Imai, and E. Masuhara, Kobunshi Ronbunshu, 35, 283 (1978).

8. S. Yamashita, S. Nagata, and K. Takakura, Kobunshi Ronbunshu, 36, 249 (1979).

9. Y. Ohtsuka, Y. Hirabayashi, J. Masubuchi, and N. Kaneko, Kobunshi Ronbunshu, 36, 257 (1979).

10. Y. Ohtsuka, Y. Hirabayashi, and S. Takayama, Kobunshi Ronbunshu, 37, 507 (1980).

11. V. T. Stannett and H. B. Hopfenberg, "Cellulose and Cellulose Derivatives," Part V, N. M. Bikales and L. Segal, Ed., Wiley-Interscience, New York, 1971, p 907.

12. S. M. Hudson and L. A. Cuculo, J. Macromol. Sci., Rev. Macromol. Chem., C18, 1 (1980).

13. M. Tsuzuki, I. Hagiwara, N. Shiraishi, and T. Yokota, J. Appl. Polym. Sci., 25, 2909 (1980).

14. N. Nishioka and K. Kosai, Polym. J., 13, 1125 (1981).

15. N. Nishioka, K. Matsumoto, and K. Kosai, Polym. J., 15, 153 (1983).

16. N. Nishioka, K. Minami, and K. Kosai, Polym. J., 15, 591 (1983).

17. D. C. Johnson, M. D. Nicholson, and F. C. Haigh, Appl. Polym. Symp., No. 28, 931 (1976).

18. S. Takigami, Y. Maeda, and Y. Nakamura, J. Appl. Polym. Sci., 24, 1419 (1979).

19. S. Takigami, Y. Maeda, and Y. Nakamura, J. Appl.
Polym. Sci., 24, 1429 (1979).

20. R. L. Cleland and W. M. Stockmayer, J. Polym. Sci., 17, 473 (1955).

21. G. Jayme and P. Kleppe, Papier, 15, 492 (1961).

22. F. de Candia, Makromol. Chem., 141, 177 (1971).

23. G. Allen, C. Booth, S. J. Hurst, M. N. Hones, and C. Price, Polymer, 8, 391 (1967).

24. J. Mann and H. J. Marrinan, Trans. Faraday Soc., 52, 492 (1956).

25. E. G. Finer, F. Franks, and M. J. Tait, J. Am. Chem. Soc., 94, 4424 (1972).

26. O. D. Bonner, J. M. Bednarek, and R. K. Arisman, J. Am. Chem. Soc., 99, 2898 (1977).

27. S. J. Wisniewski and S. W. Kim, J. Membrane Sci., 6, 299 (1980).

28. T. Uragami, Y. Sugitani, and M. Sugihara, Polymer, 23, 192 (1982).

29. S. C. Yoon and M. S. Jhon, J. Appl. Polym. Sci., 27, 3133 (1982).

30. S. Krause, "Polymer Blends," Vol. 1, D. R. Paul and S. Newman, Ed., Academic Press, New York, 1978, p 15.

31. R. E. Kesting, "Cellulose and Cellulose Derivatives," Part V, N. M. Bikales and L. Segal, Ed., WileyInterscience, New York, N. Y., 1971, p 1233.

32. J. Komiyama and R. M. Fuoss, Proc. Natl. Acad. Sci., U.S.A., 69, 829 (1972).

33. S. W. Kim, J. R. Cardinal, S. J. Wisniewski, and G. M. Zentner, "Water in Polymer," S. P. Rowland, Ed., American Chemical Society, Washington D.C., 1980, p 347.

34. D. G. Pedley and B. J. Tighe, Br. Polym. J., 11, 130 (1979).

35. G. N. Ling, "Water Structure at the Water-Polymer Interface," H. H. G. Jellinek, Ed., Plenum, New York, 1972, p 4.

36. T. Matsuura, "Gouseimaku no Kiso," Kitami Shobo, Tokyo, 1981.

37. Y. Taniguchi and S. Horigome, J. Appl. Polym. Sci., 19, 2743 (1975).

38. N. Nishioka, S. Yoshimi, T. Iwaguchi, and K. Kosai, Polym. J., 16, 885 (1984). 